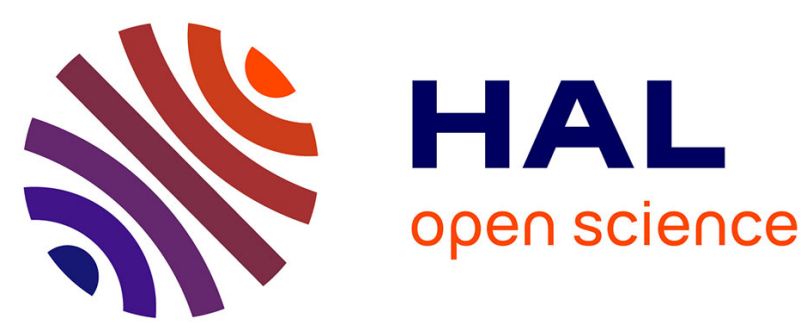

\title{
Fluid Forces on a Circular Cylinder Subjected to a Transient Motion at Low Amplitude: Infinite Medium and Cylindrical Confinement
}

Vincent Melot, Cédric Leblond, Jean-François Francois Sigrist, Christian Laine, Bruno Auvity, Hassan Peerhossaini

\section{To cite this version:}

Vincent Melot, Cédric Leblond, Jean-François Francois Sigrist, Christian Laine, Bruno Auvity, et al.. Fluid Forces on a Circular Cylinder Subjected to a Transient Motion at Low Amplitude: Infinite Medium and Cylindrical Confinement. 2006 ASME Joint US-European Fluids Engineering Division Summers Meeting, Jul 2006, Miami, FL, United States. pp.437 - 444, 10.1115/FEDSM2006-98120 . hal-01380381

\section{HAL Id: hal-01380381 https://hal.science/hal-01380381}

Submitted on 13 Oct 2016

HAL is a multi-disciplinary open access archive for the deposit and dissemination of scientific research documents, whether they are published or not. The documents may come from teaching and research institutions in France or abroad, or from public or private research centers.
L'archive ouverte pluridisciplinaire HAL, est destinée au dépôt et à la diffusion de documents scientifiques de niveau recherche, publiés ou non, émanant des établissements d'enseignement et de recherche français ou étrangers, des laboratoires publics ou privés. 


\section{FLUID FORCES ON A CIRCULAR CYLINDER SUBJECTED TO A TRANSIENT MOTION AT LOW AMPLITUDE IN INFINITE MEDIUM AND IN CYLINDRICAL CONFINEMENT}

\author{
Vincent MELOT \\ Laboratoire de Thermocinétique \\ CNRS UMR 6607 \\ Rue Christian Pauc - BP 50609 \\ 44306 Nantes, France \\ Tel : 00.33.(0)2.40.68.31.53 \\ Fax : 00.33.(0)2.40.68.31.41 \\ Email: vincent.melot@univ-nantes.fr
}

\author{
Cedric LEBLOND \\ Jean Francois SIGRIST \\ Christian LAINE \\ DCN Propulsion \\ Service Technique et Scientifique \\ 44620 La Montagne, France
}

\author{
Bruno AUVITY \\ Hassan PEERHOSSAINI \\ Laboratoire de Thermocinétique \\ CNRS UMR 6607 \\ Rue Christian Pauc - BP 50609 \\ 44306 Nantes, France
}

\section{ABSTRACT}

The present paper deals with the fluid forces induced by a rapidly moving rigid circular cylinder in an incompressible fluid initially at rest. The cylinder is subjected to an impulsive motion which corresponds to an unique sinusoidal period of acceleration and is then stopped. Two fluid domains are considered: infinite and cylindrically confined. This study focuses on small displacements of the cylinder in regards to its radius, i.e. for low Keulegan-Carpenter numbers.

In a first part, the fluid is assumed potential. Only the inner cylinder is displaced and the outer, in the confined case, is at rest. The problem, formulated as a two-dimensional boundaryperturbation problem, is solved thanks to a regular expansion. A non-linear analytical formulation of the fluid forces experienced by the moving cylinder is then proposed. Its range of validity is discussed in regards to the inner cylinder displacement. The results are confronted to numerical simulations with a CFD code based on a finite volume discretization on a moving mesh.

In a second part, a two-dimensional viscous flow is considered. Analytical formulations of the fluid forces experienced by the cylinder subjected to arbitrary motions are proposed. The starting point of the analytical approach is the fluid forces expressions obtained with an harmonic motion. These expressions come from the Rosenhead model for the infinite fluid domain. A Fourier transform is applied on the harmonic solutions to capture the wide frequency spectrum composing the transient motion. An inverse Fourier transform is then applied on the resulting expressions to derive the solutions in the temporal space. The analytical solutions are discussed and compared to numerical simulation results obtained in an infinite domain for various Stokes numbers. The competition between the viscous diffusion time and the wave duration time is studied which allow to underline history effects on the force.

\section{INTRODUCTION}

The mechanical design of naval propulsion nuclear reactors for dynamic imposed loading must take into account fluidstructure interaction phenomena. A global R\& D approach has been developed by DCN Propulsion for industrial applications. In a first step, numerical simulation techniques for the coupled fluid-structure problem were investigated [1]. A second step is now undertaken within a collaborative research program between DCN PROPULSION and LABORATOIRE DE THERMOCINÉTIQUE DE NANTES in order to study the fluid forces acting on a moving body and to describe the physical phenomena occurring in the fluid domain. The topic of this collaboration is centered on a cylinder impulse in cylindrical confinement. The inner cylinder is subjected to an impulsive motion which corresponds to an unique sinusodal period of acceleration and is then 
stopped :

$$
\left\{\begin{array}{l}
\ddot{e}(t)=\ddot{e}_{M A X} \sin \left(\omega_{0} t\right) \quad \forall t \in\left[0, T_{0}[\right. \\
\ddot{e}(t)=0 \quad \forall t \in\left[T_{0}, \infty[\right.
\end{array}\right.
$$

where $e(t)$ is the inner cylinder displacement, $T_{0}$ is the shock duration, $\omega_{0}$ is the pulsation defined by $\omega_{0}=2 \pi / T_{0}$. The Figure 1 presents the normalized acceleration $\ddot{e}(t) / \ddot{e}_{M A X}$, the normalized velocity $\dot{e}(t) / \dot{e}_{M A X}$ and the normalized displacement $e(t) / e_{M A X}$ in function of the dimensionless time $t^{*}$ defined by $t^{*}=t / T_{0}$ :

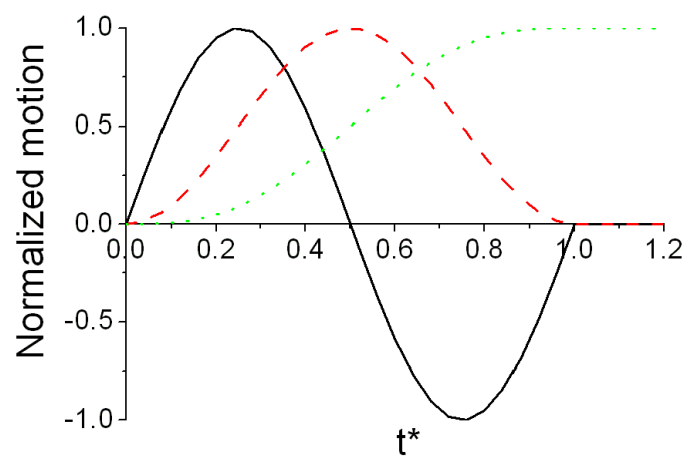

Figure 1. Normalized acceleration, velocity and displacement. $\ddot{e}(t) / \ddot{e}_{M A X} ;--\dot{e}(t) / \dot{e}_{M A X} ; \ldots e(t) / e_{M A X}$.

The system can be characterized by at least three dimensionless numbers, the Stokes number, the Keulegan-Carpenter number and the confinement ratio $\alpha$ :

$$
\beta=\frac{D_{1}^{2}}{T_{0} v} \quad K C=\frac{e_{M A X}}{D_{1}} \quad \alpha=\frac{R_{2}}{R_{1}}
$$

where $D_{1}$ and $D_{2}$ are respectively the diameters of the inner and the outer cylinder, $R_{1}$ and $R_{2}$ their radius and $v$ the kinematic viscosity. The Stokes number compares the viscous diffusion time $D_{1}^{2} / \mathrm{v}$ to the impulse time $T_{0}$. The Keulegan-Carpenter number compares the maximum displacement of the cylinder with its diameters and the ratio $\alpha$ quantifies the geometrical confinement. Another dimensionless number $\xi$ will be used in this paper:

$$
\xi=\frac{e_{M A X}}{R_{2}-R_{1}}
$$

It allows to quantify the inner cylinder displacement in regards to the radial clearance.

Complex phenomena can potentially arise in this system, like boundary layer separations [2] due to large amplitude motions of the inner cylinder, three dimensional instabilities [3] or cavitation. Here the problem is assumed two dimensional and only low Keulegan-Carpenter numbers are considered. Then, separation mechanisms are not expected to occur. Furthemore three dimensional instabilities are not present. By taking into account these assumptions, there remain two phenomena being able to appear in this problem, the confinement and viscous effect. Then, the first part of this paper is interested by the effect of confinement in inviscid fluid. In a second paper, the problem of viscous flow in infinite domain is tackled. For each of the two problems, an analytical formulation of fluid forces is proposed and compared with numerical simulation.

The numerical code [4] is based on a second-order finite volume discretization scheme. The Navier-Stokes equations are written in their general conservative form [5] with an arbitrary lagrangian eulerian formulation [6]. Hence moving boundaries can be taken into account. The PISO algorithm [7] is used to handle the coupling between pressure and velocity. The computational domain used for the numerical simulations is represented in Figure 2 for the infinite case. This domain is divided into three regions: an upstream region (at the left hand side of the domain), a downstream region (at the left hand side of the domain) constituted with large square cells and a square region (in middle of the computational domain) with a refined mesh close to the cylinder wall. This last is composed of four parts surrounding an annular space. When the inner cylinder is in motion, only the annular space of the computational domain is deformed. For the confined case, only the annular space is kept. The numerical code was validated for a Dirac acceleration in a previous paper [8].

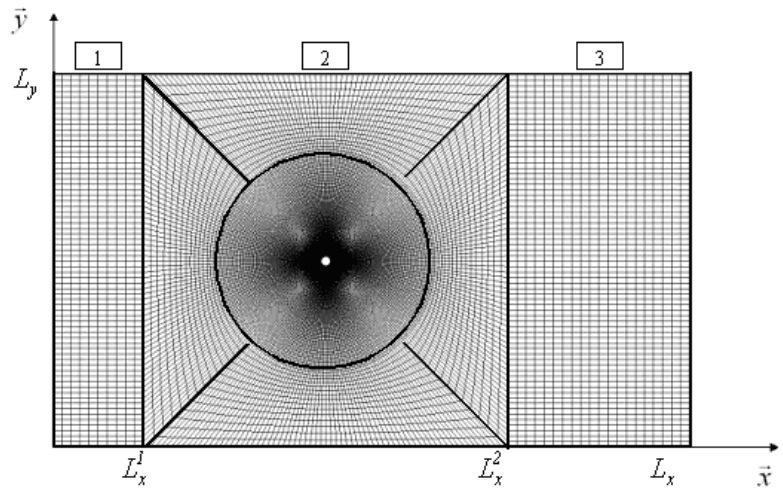

Figure 2. Mesh grid of the computational domain.

\section{INVISCID PROBLEM IN CONFINED FLOWS}

\subsection{Problem formulation}

In this part, a rapidly moving circular cylinder in a annular fluid region is considered. One of the most currently used model available in the literature related to this geometry is the Fritz one [9], where the following assumptions are made: the flow is two-dimensional, incompressible, inviscid and initially at rest. Moreover, the displacement $e(t)$ imposed to the inner cylinder is supposed very small compared to its radius $\left(e(t) / R_{1}<<1\right)$. Hence advection terms and geometrical deformations are also neglected. In this part, quite large displacements are considered, so that the advection term and the geometrical deformation have to be taken into account. However the inner cylinder displacement is assumed sufficiently small so that no separation occurs and rapid enough to produce boundary layers [10] whose thickness are much thinner than the radial clearance $\left(\sqrt{v T_{0}}<<R_{2}-R_{1}\right)$ 


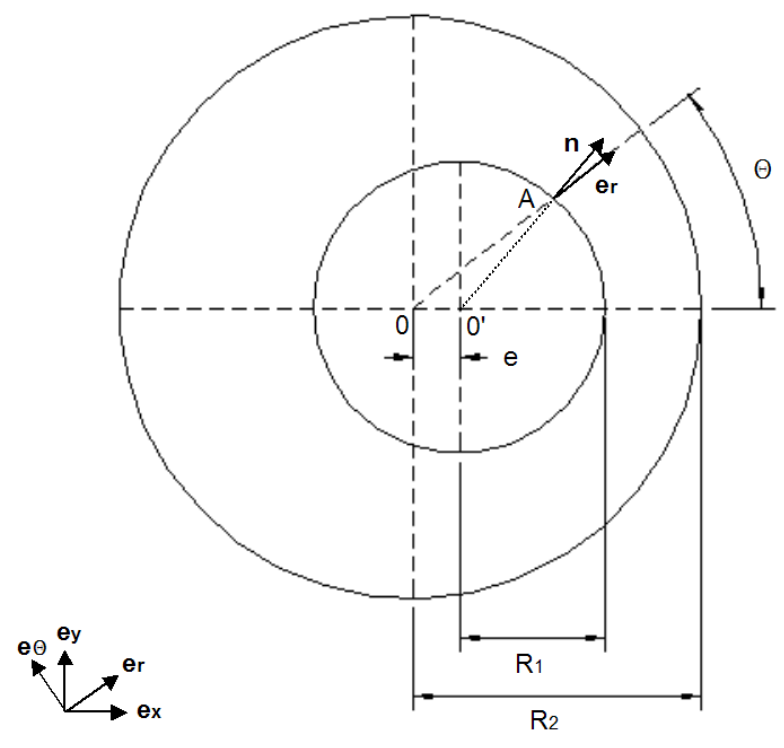

Figure 3. Geometrical configuration.

$[11,12]$. The problem is formulated as a boundary-perturbation problem [13] for the velocity potential and a regular expansion is used to solve it up to the second order. The integrated forces are then given and compared with those obtained from a CFD code [4] based on a finite-volume discretization on a moving mesh. Another way of thinking large displacements effects can be found in $[14,15]$. Since the flow is assumed inviscid, the Navier-Stokes equations governing the fluid dynamics are reduced to:

$$
\begin{aligned}
\frac{\partial \mathbf{u}}{\partial t}+(\mathbf{u} \cdot \nabla) \mathbf{u} & =-\frac{1}{\rho} \nabla p \\
\nabla \cdot \mathbf{u} & =0
\end{aligned}
$$

The fluid is also assumed initially irrotational, so it remains irrotational at latter times and there exists a function $\Phi$, called the velocity potential, such that $\mathbf{u}=\nabla \Phi$. Using the continuity equation Eqn. (5), it comes that $\Phi$ satisfies the Laplace equation in the fluid domain:

$$
\nabla^{2} \Phi=0
$$

This derivation is classical and can be found in all fluid dynamics books [16]. Since the fluid is assumed inviscid, only the normal component of the velocity has to be conserved on the solid boundaries. For the geometry of interest in this paper and given in Fig. 3, the boundary conditions in term of velocity potentiel are:

$$
\begin{aligned}
\nabla \Phi \cdot \mathbf{e}_{\mathbf{r}} & =0 \quad \text { on the fixed outer cylinder } \\
\nabla \Phi \cdot \mathbf{n} & =\dot{e}(t) \mathbf{e}_{\mathbf{x}} \cdot \mathbf{n} \quad \text { on the moving inner cylinder }
\end{aligned}
$$

In order to make this problem analytically tractable, the inner cylinder position and the unit outward normal $\mathbf{n}$ have to be expressed in explicit terms in Eqn. (8). By considering the triangle
$O O^{\prime} A$ in Fig. 3, it is straightforward to find that the inner circular cylinder position $r_{c}$ satisfies the following relation:

$$
R_{1}^{2}=r_{c}^{2}+e^{2}(t)-2 r_{c} e(t) \cos \theta
$$

Since we are interested by motions of the inner cylinder much smaller than its radius, the physical solution of Eqn. (9) is:

$$
r_{c}(\theta, t)=e(t) \cos \theta+R_{1} \sqrt{1-\frac{e^{2}(t)}{R_{1}^{2}} \sin ^{2} \theta}
$$

This is the polar equation of the inner cylinder $C(t)$. Expanding the square root in terms of series gives

$$
\begin{aligned}
r_{c}(\theta, t) & =R_{1}\left(1+\cos \theta \frac{e(t)}{R_{1}}\right. \\
& \left.+\sum_{n=1}^{\infty}(-1)^{n}(\sin \theta)^{2 n} \frac{1}{n !} \prod_{k=0}^{n-1}\left(\frac{1}{2}-k\right)\left(\frac{e(t)}{R_{1}}\right)^{2 n}\right)
\end{aligned}
$$

which always converges for $\left|e(t) / R_{1}\right|<1$. Thanks to this formula, we define approximate positions of the inner cylinder:

$$
\begin{aligned}
& r_{0}(\theta)=R_{1} \\
& r_{1}(\theta)=R_{1}\left(1+\cos \theta \frac{e(t)}{R_{1}}\right) \\
& r_{2}(\theta)=R_{1}\left(1+\cos \theta \frac{e(t)}{R_{1}}-\frac{1}{2} \sin ^{2} \theta\left(\frac{e(t)}{R_{1}}\right)^{2}\right)
\end{aligned}
$$

The boundary condition Eqn. (8) will be expressed thanks to this formula. $r_{0}$ is used in the Fritz model [9] and is the leading order approximation of Eqn. (11). $r_{1}$ and $r_{2}$, respectivily the first and second order approximations of Eqn. (11), will be used to locate $C(t)$ in the first order and second order models. It is also of interest to write in explict terms the unit outward normal $\mathbf{n}$ on the moving inner cylinder. We consider for this the parametric curve $\Psi$ of $C(t)$ which is defined by:

$$
\mathcal{C}(t): \theta \mapsto \Psi(\theta)=O+r_{c}(\theta, t) \mathbf{e}_{\mathbf{r}}(\theta, t)
$$

where $O$ is the centre of the outer cylinder. The unit tangent $\mathbf{T}$ to $\mathcal{C}(t)$ at the position $\theta$ is given by:

$$
\mathbf{T}(\theta)=\frac{\Psi^{\prime}(\theta)}{\left\|\Psi^{\prime}(\theta)\right\|}
$$

where $\Psi^{\prime}(\theta)$ and $\left\|\Psi^{\prime}(\theta)\right\|$ can be written:

$$
\begin{aligned}
\Psi^{\prime}(\theta) & =r_{c}^{\prime}(\theta) \mathbf{e}_{\mathbf{r}}+r_{c}(\theta) \mathbf{e}_{\theta} \\
\left\|\Psi^{\prime}(\theta)\right\| & =\sqrt{r_{c}^{\prime 2}(\theta)+r_{c}^{2}(\theta)}
\end{aligned}
$$

and where the prime denotes derivative according to $\theta$. The unit normal $\mathbf{n}$ which is orthogonal to $\mathbf{T}$ can then be evaluated thanks to Eqns. (12-17). Its truncation at the leading, first and second 
orders are respectively:

$$
\begin{aligned}
\mathbf{n}_{\mathbf{0}}(\theta) & =\frac{1}{\left\|\Psi^{\prime}(\theta)\right\|}\left(R_{1} \cos \theta \mathbf{e}_{\mathbf{x}}+R_{1} \sin \theta \mathbf{e}_{\mathbf{y}}\right) \\
\mathbf{n}_{\mathbf{1}}(\theta) & =\frac{1}{\left\|\Psi^{\prime}(\theta)\right\|}\left(R_{1} \cos \theta+e(t) \cos 2 \theta\right) \mathbf{e}_{\mathbf{x}} \\
& +\frac{1}{\left\|\Psi^{\prime}(\theta)\right\|}\left(R_{1} \sin \theta+e(t) \sin 2 \theta\right) \mathbf{e}_{\mathbf{y}} \\
\mathbf{n}_{\mathbf{2}}(\theta) & =\frac{1}{\left\|\Psi^{\prime}(\theta)\right\|}\left(\left(R_{1}-\frac{3}{8} \frac{e^{2}(t)}{R_{1}}\right) \cos \theta\right. \\
& \left.+e(t) \cos 2 \theta+\frac{3}{8} \frac{e^{2}(t)}{R_{1}} \cos 3 \theta\right) \mathbf{e}_{\mathbf{x}} \\
& +\frac{1}{\left\|\Psi^{\prime}(\theta)\right\|}\left(\left(R_{1}-\frac{3}{8} \frac{e^{2}(t)}{R_{1}}\right) \sin \theta\right. \\
& \left.+e(t) \sin 2 \theta+\frac{3}{8} \frac{e^{2}(t)}{R_{1}} \sin 3 \theta\right) \mathbf{e}_{\mathbf{y}}
\end{aligned}
$$

In order to evaluate the fluid forces on the inner cylinder, the knowledge of $\mathbf{n} d s$ is also required. $d s$ is an infinitesimal element of the curviligne abscissa of $C(t)$ and is given by the formula:

$$
d s=\left\|\Psi^{\prime}(\theta)\right\| d \theta
$$

Hence the expression of $\mathbf{n} d s$ can be directly deduced from Eqns. $(19,20,21)$. It is now convenient to rewrite the system governing the velocity potential Eqns. $(6,7,8)$ in polar coordinates:

$$
\frac{\partial^{2} \Phi}{\partial r^{2}}+\frac{1}{r} \frac{\partial \Phi}{\partial r}+\frac{1}{r^{2}} \frac{\partial^{2} \Phi}{\partial \theta^{2}}=0
$$

for $(r, \theta) \in] r_{c}(\theta), R_{2}[\times[0,2 \pi[$ and:

$$
\begin{aligned}
\frac{\partial \Phi}{\partial r}\left(R_{2}, \theta\right) & =0 \\
\nabla \Phi\left(r_{c}, \theta\right) \cdot \mathbf{n}\left(r_{c}, \theta\right) & =\dot{e}(t) \mathbf{e}_{\mathbf{x}} \cdot \mathbf{n}\left(r_{c}, \theta\right)
\end{aligned}
$$

on the boundaries. Hence the system of equations to solve is a laplacian with Neumann boundary condition on the outer cylinder. A difficulty arises from the boundary condition on the inner cylinder since $r_{c}$ and $\mathbf{n}$ are functions of $\theta$ and $t$. Since there is no differentiation with time in this system, $t$ is only a parameter. Equation (25) is seen as the extreme boundary condition in the following family of boundary conditions:

$$
\nabla \Phi\left(r_{n}(\theta), \theta\right) \cdot \mathbf{n}\left(r_{n}(\theta), \theta\right)=\dot{e}(t) \mathbf{e}_{\mathbf{x}} \cdot \mathbf{n}\left(r_{n}(\theta), \theta\right)
$$

where $r_{n}(\theta)$ takes the form:

$$
r_{n}(\theta)=\sum_{p=0}^{n} A_{p} \varepsilon^{p}
$$

and satisfies:

$$
\lim _{n \mapsto \infty} r_{n}(\theta)=r_{c}(\theta) .
$$

The perturbation parameter $\varepsilon$ is in our case $e(t) / R_{1}$ and the coefficients $A_{p}$ can be identified by considering Eqn. (11). Performing a Taylor expansion of Eqn. (25) about $R_{1}$ and using the de- composition Eqn. (27) allow to turn the original problem into an equivalent one. We can now divide the problem into a sequence of problems where we can separately find the functions $\Phi_{0}, \Phi_{1}$, $\Phi_{2}$ :

$$
\Phi=\sum_{n=0}^{\infty}\left(\frac{e(t)}{R_{1}}\right)^{n} \Phi_{n}
$$

If the perturbation parameter is sufficiently small, the serie will converge rapidly and few terms will be sufficient to provide a good approximation of the solution. In this paper, only the main order $\Phi_{0}$, first order $\Phi_{1}$ and second order $\Phi_{2}$ approximations are found. Hence the solution is expected to be valid in cases where $\left(e(t) / R_{1}\right)^{3}<<1$. Once the velocity potential is found, the pressure is obtained thanks to the Bernoulli equation [16]:

$$
p(r, \theta)=-\rho \frac{\partial \Phi}{\partial t}(r, \theta)-\rho \frac{1}{2} \mathbf{u}^{2}(r, \theta)+C
$$

where $C$ is a constant available in the whole fluid domain. Since the flow is assumed inviscid, integrated fluid forces on the moving inner circular cylinder are given by:

$$
\varphi(t)=-\int_{0}^{2 \pi} p(r(\theta)) \overline{\bar{I}} \cdot \mathbf{n}(\theta)\left\|\Psi^{\prime}(\theta)\right\| d \theta
$$

where $\overline{\bar{I}}$ is the identity matrix. In the next section, the problem is analytically solved at the leading, first and second orders.

\subsection{Leading, first and second order resolutions}

By introducing the decomposition Eqn.(29) in the linear Laplace equation Eqn.(23) and in the outer cylinder boundary condition Eqn.(24), it can be inferred that each component of the decomposition satisfies the Laplace equation in the fluid domain and a Neumann boundary condition on the outer cylinder. The boundary conditions on the inner cylinder are found by introducing the decomposition Eqn.(29) in Eqn.(25) and by using the leading, first and second order approximations of $r_{c}$ and $\mathbf{n}$ given respectively by Eqns. $(12,13,14)$ and Eqns. $(19,20,21)$. For the leading-order approximation it gives:

$$
\frac{\partial \Phi_{0}}{\partial r}\left(r_{0}, \theta\right)=\dot{e}(t) \cos \theta
$$

Hence, the leading-order problem consists in solving a Laplacian with Neumann boundary conditions in an annular geometry. It has been already solved by Fritz [9] by the method of separation of variables, which results in:

$$
\Phi_{0}(r, \theta)=-\frac{1}{\alpha^{2}-1}\left(r+\frac{R_{2}^{2}}{r}\right) \dot{e}(t) \cos \theta+B_{0}
$$

The knowledge of $\Phi_{0}$ allows us to write the first-order boundary condition on the inner cylinder thanks to Taylor series expansion at $r=R_{1}$ in explicit terms:

$$
\frac{\partial \Phi_{1}}{\partial r}\left(R_{1}, \theta\right)=\frac{2 \alpha^{2}}{\alpha^{2}-1} \dot{e}(t) \cos 2 \theta
$$

The first order problem consists also in solving a Laplace equation in an annular fluid region with Neumann boundary condi- 
tions. With the method of separation of variable, the solution is found:

$$
\Phi_{1}(r, \theta)=\frac{-\alpha^{2}}{\left(\alpha^{2}-1\right)\left(\alpha^{4}-1\right)} \dot{e}(t) \frac{R_{2}^{2}}{R_{1}}\left(\frac{r^{2}}{R_{2}^{2}}+\frac{R_{2}^{2}}{r^{2}}\right) \cos 2 \theta
$$

Once again, the knowledge of $\Phi_{0}$ and $\Phi_{1}$ allow us to express the second order approximation boundary condition on the inner cylinder thanks to Taylor series expansion. After some manipulations it takes the form:

$$
\begin{aligned}
\frac{\partial \Phi_{2}}{\partial r}\left(R_{1}, \theta\right) & =\frac{2 \alpha^{2}}{\left(\alpha^{2}-1\right)\left(\alpha^{4}-1\right)} \dot{e}(t) \cos \theta \\
& +\frac{3 \alpha^{2}\left(\alpha^{4}+1\right)}{\left(\alpha^{2}-1\right)\left(\alpha^{4}-1\right)} \dot{e}(t) \cos 3 \theta
\end{aligned}
$$

Hence the second order problem is again a Laplace equation with Neumann boundary conditions and the solution can be written:

$$
\begin{aligned}
\Phi_{2}(r, \theta)= & -\dot{e}(t) \frac{2 \alpha^{2}}{\left(\alpha^{2}-1\right)^{2}\left(\alpha^{4}-1\right)}\left(r+\frac{R_{2}^{2}}{r}\right) \cos \theta \\
& -\dot{e}(t) \frac{\alpha^{2}\left(\alpha^{4}+1\right)}{\left(\alpha^{2}-1\right)\left(\alpha^{4}-1\right)\left(\alpha^{6}-1\right)} \frac{1}{R_{1}^{2}}\left(r^{3}+\frac{R_{2}^{6}}{r^{3}}\right) \cos 3 \theta
\end{aligned}
$$

The leading, first and second order approximations of the pressure field in the fluid domain can then be deduced thanks to the Bernoulli equation Eqn.(30). By evaluating the obtained pressure fields on the inner cylinder position $r=r_{c}$ using Taylor series expansion around $r=R_{1}$, and by integrating thanks to the formulae Eqns.(31,19,20,21), the integrated pressure forces can be obtained. Only the components in the $\mathbf{e}_{\mathbf{x}}$-direction non zero. The leading order expression is

$$
\varphi_{0}(t)=-\rho \pi R_{1}^{2} \ddot{e}(t) \frac{\alpha^{2}+1}{\alpha^{2}-1}
$$

which is exactly the Fritz model expression [9]. Since it is in proportion with the acceleration, it consists only in an added mass effect. For an infinite fluid domain $(\alpha \rightarrow \infty)$ it reduces to $\varphi_{0}(t)=-\rho \pi R_{1}^{2} \ddot{e}(t)$ which is the well-known fluid displaced effect. The first order approximation is given by

$$
\begin{aligned}
\varphi_{1}(t) & =-\rho \pi R_{1}^{2} \ddot{e}(t) \frac{\alpha^{2}+1}{\alpha^{2}-1} \\
& +\rho \pi e(t) \dot{e}^{2}(t) \frac{2 \alpha^{2}\left(\alpha^{2}+1\right)}{\left(\alpha^{2}-1\right)^{2}\left(\alpha^{4}-1\right)}+O\left(\frac{e(t)}{R_{1}}\right)^{2}
\end{aligned}
$$

A new term has appeared in the right hand side of the above formula. It can be inferred that the advection term which does not influence the fluid force at the leading-order, modify the integrated pressure force at the first order. The second order expres- sion can be written after some manipulations

$$
\begin{aligned}
\varphi_{2}(t) & =-\rho \pi R_{1}^{2} \ddot{e}(t) \frac{\alpha^{2}+1}{\alpha^{2}-1} \\
& +\rho \pi e(t) \dot{e}^{2}(t) \frac{2 \alpha^{2}\left(\alpha^{2}+1\right)}{\left(\alpha^{2}-1\right)^{2}\left(\alpha^{4}-1\right)} \\
& -\rho \pi e^{2}(t) \ddot{e}(t) \frac{4 \alpha^{4}}{\left(\alpha^{2}-1\right)^{2}\left(\alpha^{4}-1\right)}+O\left(\frac{e(t)}{R_{1}}\right)^{3}
\end{aligned}
$$

A new term appears again. It can be seen as a non linear rectification of the added mass coefficient. Validity and limits of these fluid forces expressions are compared with numerical simulation results in the next section.

\subsection{Comparaison of the results with numerical simu- lations}

The inviscid analytical model is only tested on its ability to take advection and geometrical deformation effects into account. Introduction of the fluid viscosity is the topic of a work currently in progress so it will not be considered here. In order to compare the results issued from numerical simulations with the simple models developped here, the motion given in Equation Eqn.(1) is imposed to the inner cylinder. We will specialy consider the confinement $\alpha=2$ for illustration but the same phenomena occur for $\alpha=1.5$ or $\alpha=1.1$. The results for different $\xi(0.1,0.6$ and $0.9)$ are computed so as to investigate the influence of large displacements in regards to the radial clearance. For each case, the numerical results have been checked to be independent of mesh refinements. The time history of the integrated fluid forces obtained with CFD simulations are compared to those predicted by the Fritz model (which corresponds to the leading-order formula Eqn.(39)) and the second order model (given in Eqn.(41)). The force calculated for the three previous models are non dimensionalized by the maximum force obtained with the Fritz model (Eqn.(39)) and are presented in Fig. 4 for $\xi=0.1 ; 0.6 ; 0.9$. For small amplitudes of the inner cylinder $(\xi=0.1)$, the fluid forces predicted by the Fritz model, the second order model and the numerical simulation are the same at each time. Increasing $\xi$, both the second order model and the numerical simulation predict bigger maximum forces than the Fritz model. However a net difference can be seen: the second order model is for each time bigger than the Fritz model, whereas numerical simulations predict smaller forces during parts of the motion. This behaviour is all the more pronounced as $\xi$ tends to 1 . For very small displacements, the Fritz model is sufficient to estimate the inviscid fluid forces. For larger ones (up to $\xi=0,6$ ), the second order model give an accurate upper bound of the fluid forces. For very high geometrical deformations $(\xi=0,9)$ both the Fritz model and the second order one underestimate the fluid forces. 

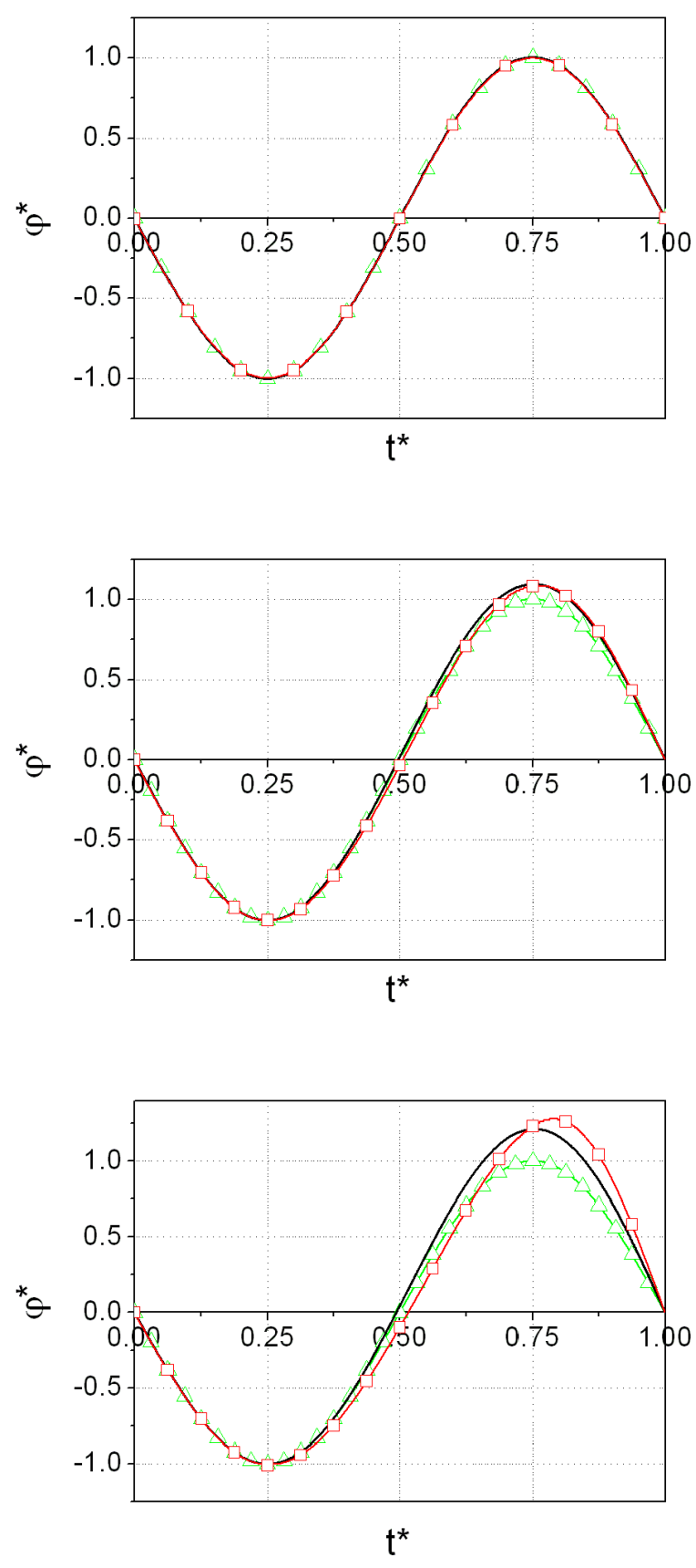

Figure 4. Normalized fluid force $\varphi^{*}$ in function of the reduced time $t^{*}$ for $\xi=0.1 ; 0.6 ; 0.9$. $\triangle$ Fritz model ; $\square$ CFD Simulation ; - Order 2 .

\section{VISCOUS PROBLEM IN INFINITE FLUID DOMAIN}

\subsection{Problem formulation}

The method proposed here is to write a general formulation of forces for any small-amplitude motions imposed to the cylinder. This formulation will then be applied to the particular movement Eqn.(1). This method consists of obtaining a general formulation of forces from a solution developed for an harmonic motion. In fact, a Fourier transform is applied on an harmonic solution to capture the wide frequency spectrum composing the transient motion. Then an inverse Fourier transform is applied on the previous expression to derive the solution in the temporal space. A review of literature [17] of fluid forces exerting on body subjected to small amplitude harmonic motions in an incompressible viscous fluid at rest allows to express the fluid forces in the form:

$$
\varphi^{H}=-M_{D}\left[(1+h) \ddot{e}(t)+h^{\prime} \omega \dot{e}(t)\right]-M_{D}\left[h \ddot{e}(t)+h^{\prime} \omega \dot{e}(t)\right]
$$

with $M_{D}=\rho \pi R_{1}^{2}$ is a displaced mass term, $h$ and $h^{\prime}$ are the coefficients depending on the pulsation $\omega$ and the kinematic viscosity $v$. The first bracket is the pressure force and the second is the shear force. The following definition of the Fourier transform of a function $f(t)$ is used [18]:

$$
\mathcal{F}\{f(t)\}=\widehat{f}(\omega)=\int_{-\infty}^{+\infty} e^{-i \omega t} f(t) d t
$$

and the inverse transform is:

$$
\mathcal{F}^{-1}\{\widehat{f}(\omega)\}=f(t)=\frac{1}{2 \pi} \int_{-\infty}^{+\infty} e^{i \omega x} \widehat{f}(\omega) d \omega
$$

Applying these definitions to the formula Eqn.(42) gives:

$$
\begin{aligned}
\widehat{\varphi}(\omega)= & -M_{D}\left[\frac{1}{N-1} \int_{-\infty}^{\infty} e^{i \omega t} \ddot{e}(t) d t+\int_{-\infty}^{\infty} e^{i \omega t} h(\omega) \ddot{e}(t) d t\right. \\
& \left.+i \int_{-\infty}^{\infty} e^{i \omega t} h^{\prime}(w) \ddot{e}(t) d t\right]-M_{D}(N-1)\left[\int_{-\infty}^{\infty} e^{i \omega t} h(\omega) \ddot{e}(t) d t\right. \\
& \left.+i \int_{-\infty}^{\infty} e^{i \omega t} h^{\prime}(w) \ddot{e}(t) d t\right]
\end{aligned}
$$

and in going back in space temporal, an equation for an arbitrary movement is obtained:

$$
\begin{aligned}
\varphi^{Q}(t)= & -M_{D}\left[\frac{1}{N-1} \int_{0}^{t} \delta(t-\tau) \ddot{e}(\tau) d \tau+\int_{0}^{t} \widetilde{h}(t-\tau) \ddot{e}(\tau) d \tau(46)\right. \\
& \left.+\int_{0}^{t} \widetilde{h}^{\prime}(t-\tau) \ddot{e}(\tau) d \tau\right]-M_{D}(N-1)\left[\int_{0}^{t} \widetilde{h}(t-\tau) \ddot{e}(\tau) d \tau\right. \\
& \left.+\int_{0}^{t} \widetilde{h}^{\prime}(t-\tau) \ddot{e}(\tau) d \tau\right]
\end{aligned}
$$

with the inverses transforms $\widetilde{h}$ et $\widetilde{h^{\prime}}$ respectively to $h$ et $h^{\prime}, \delta$ is the Dirac function and $\tau$ a variable. Now, it is necessary to search a solution in harmonic motion to achieve the inverse coefficients $\widetilde{h}$ and $\widetilde{h^{\prime}}$. STOKES [19] writes the coefficients $h$ and $h^{\prime}$ under this following forms:

$$
h-i h^{\prime}=\frac{2}{\lambda R_{1}} \frac{K_{1}\left(\lambda R_{1}\right)}{K_{0}\left(\lambda R_{1}\right)}
$$

with $\lambda=\sqrt{\frac{i \omega}{v}} . K_{1}$ and $K_{0}$ are related the Bessel modified of second species respectively to the order 1 and 0 [18]. However, it is difficult to find the inverse $\widetilde{h}$ and $\widetilde{h^{\prime}}$ because the relation 47 is complex. Indeed, it presents a singularity when $\lambda R_{1}$ tends to 
zero and it is not possible to realize simply a FFT on this relation to obtain the inverses. An other solution is to obtain the inverses with a approach model. It exists the Rosenhead model [20] written under the form of successive approximation:

$$
\left\{\begin{array}{l}
h=\frac{1}{R_{1}} \sqrt{\frac{2 v}{\omega}} \\
h^{\prime}=\frac{1}{R_{1}} \sqrt{\frac{2 v}{\omega}}+\frac{1}{R_{1}^{2}} \frac{v}{\omega}
\end{array}\right.
$$

This model is close to the Stokes model for any value of the Stokes number [17]. The inverse transform of $1 / \sqrt{\omega}$ and $1 / \omega$ are respectively $1 / \sqrt{2 \pi t}$ and 1 [18]. The relation Eqn.(46) takes then the following form:

$$
\begin{aligned}
\varphi^{Q} & =-M_{D}\left[\ddot{e}(t)+\frac{2}{R_{1}} \sqrt{\frac{\nu}{\pi}} \int_{0}^{t} \frac{\ddot{e}(\tau)}{\sqrt{t-\tau}} d \tau+\frac{v}{R_{1}^{2}} \dot{e}(t)\right] \\
& -M_{D}\left[\frac{2}{R_{1}} \sqrt{\frac{\nu}{\pi}} \int_{0}^{t} \frac{\ddot{e}(\tau)}{\sqrt{t-\tau}} d \tau+\frac{\nu}{R_{1}^{2}} \dot{e}(t)\right]
\end{aligned}
$$

The first term of pressure force is the displaced mass term. The second term of the pressure force and the first of the shear force is the history term and the last term of each forces is the drag term. This model will be called "general model". With the motion described in equation Eqn.(1), the general model is reduced to:

$$
\begin{aligned}
\varphi= & -M_{D} \ddot{e}_{M A X}\left[\sin \left(2 \pi t^{*}\right)+\frac{8}{\sqrt{\pi}} \beta^{-1 / 2} \sqrt{T_{0}} \int_{0}^{t} \frac{\sin \left(2 \pi \tau / T_{0}\right)}{\sqrt{t-\tau}} d \tau+\right. \\
& \left.\frac{4}{\pi} \beta^{-1}\left(1-\cos \left(2 \pi t^{*}\right)\right)\right]
\end{aligned}
$$

When $\beta$ becomes very large, this model tends to the Fritz model validated for infinite domain.

\subsection{Comparaison of the results with numerical simu- lations}

These models are validated for any STOKES number, $\beta=$ $10 ; 100 ; 20,000$ for low value of $K C,(K C=0.0005)$ respecting the small displacements assumption. The figure Fig. 5 presents respectively the force $\varphi^{*}$ in function of reduced time $t^{*}$ during and after the impulse with numerical simulation (CFD Model) and analytical formulation (general model). The forces $\varphi^{*}$ are non dimensionalized by the approached maximal force:

$$
\left|\varphi^{Q}\right|=-M_{D} \ddot{e}_{M A X}\left[1+\frac{8}{\sqrt{\pi}} \beta^{-1 / 2}+\frac{8}{\pi} \beta^{-1}\right]
$$

For any STOKES number, the CFD model and General model gives same results, less than 1\%. For high STOKES number, the three models tend toward the same trend. The force is in proportion to the acceleration of the cylinder according the formula Eqn.(50). The fluid can hence be considered as inviscid. The viscous terms in Eqn.(50) are negligible in regards to the inertia term. For low value of STOKES number, the general and CFD models differs from the Fritz model. Indeed, the maxi-
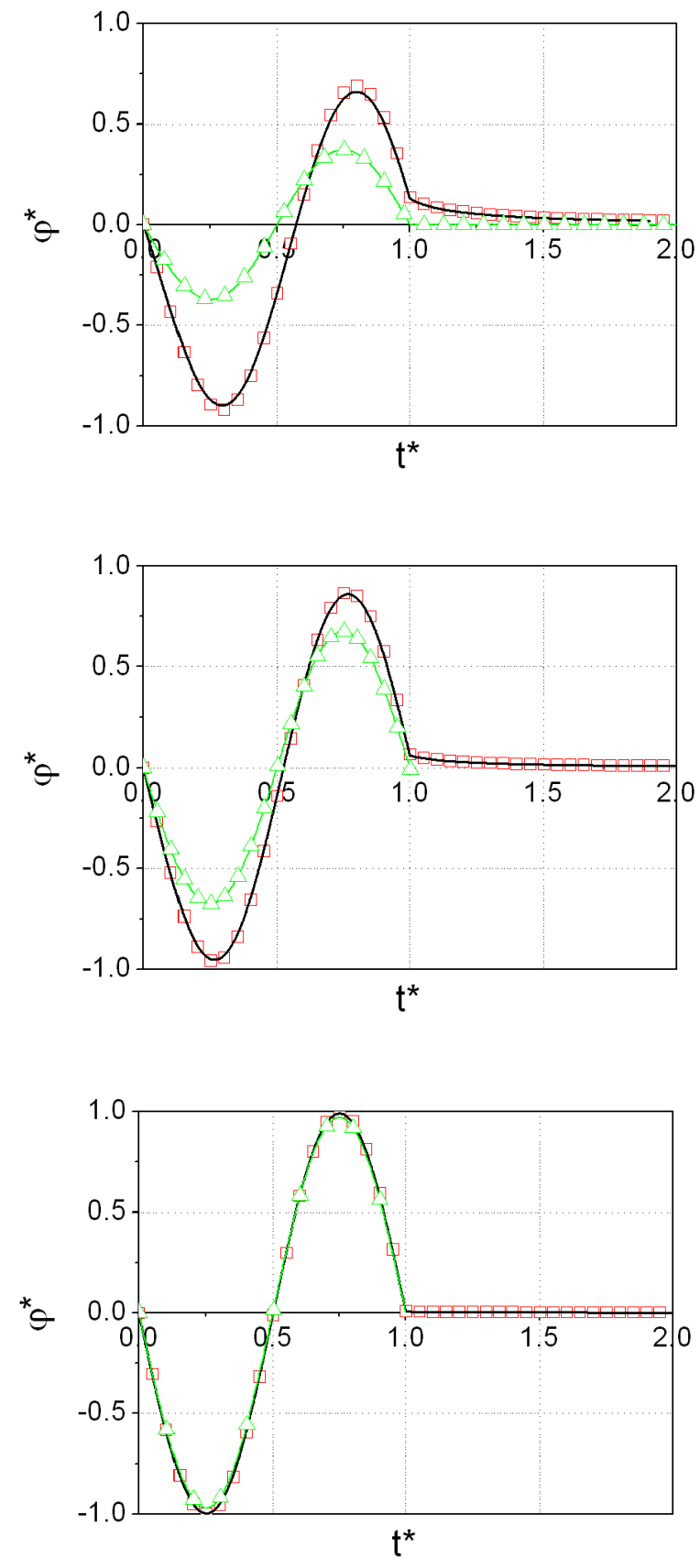

Figure 5. Normalized fluid force $\varphi^{*}$ in function of the reduced time $t^{*}$ for $\beta=10 ; 100 ; 20,000$. $\triangle$ Fritz model ; $\square$ CFD Simulation ; - General model.

mum force of the two previous models are higher than the Fritz model, approximatively 2.5 more higher in considering the formula Eqn.(51). Moreover, the force for general and CFD models are not exactly proportional to the acceleration imposed to the cylinder. The force are not canceled with $t^{*}=0.5$ but for larger 
values and the positive maximum force $\left|+\varphi^{*}\right|$ are not always equal to the negative maximum force $\left|-\varphi^{*}\right|$ on the range of the Stokes number. Furthemore, it exists a residual force after the shock, i.e. for $t^{*}>1$ for general model and CFD model. These changes at low values of $\beta$ are due to due the viscosity effects. Indeed, for small $\beta$ numbers, the three terms of the equation (50) are in competition, especially between the inertia term and the history term. The drag term is a second order term, it does not bring further understanding of the solution. The history term permits to take into account the whole transitional stage of the movement in the calculation of the fluid force.

\section{CONCLUSION}

For a confined inviscid flow, extensions of the Fritz model are performed by taking advection terms and the geometrical deformations induced by the inner circular cylinder movement into account. Approximated analytical solutions are found with a regular expansion performed until the second order on a boundaryperturbation problem. The resulting fluid forces are then compared to numerical simulation predictions performed with a code able to take into account moving fluid domains. The second order model is shown to be more efficient than the Fritz model, specialy for high geometrical deformation. Nevertheless these two models are not able to reproduce strongly non linear potential effects found with the numerical simulations for high inner cylinder displacements.

In infinite fluid domain, an analytical model, called "general model" is proposed to take into account the viscous effect. These last is compared with success to the CFD simulations and shows discrepancies with Fritz model. For high Stokes numbers, these models tend to inviscid case whereas for small Stokes numbers, a general model and CFD simulations permit to highlight the history effect due the fluid viscous.

Future extensions of the presented work in the confined case would include viscous effects in order to characterize the damping term in the fluid forces like the infinite case. For the infinite fluid domain, an investigation would be performed for large displacement of the cylinder corresponding to high KeuleganCarpenter numbers to explore the non linear effect of advection.

\section{REFERENCES}

[1] J.F. Sigrist, C. Laine, D. Lemoine, and B. PeseuX. Choice and limits of a linear fluid model for the numerical study in fluid structure interaction problem. In ASME Pressure Vessel and Piping Div. Conf, Cleveland, USA, 20-24 July 2003.

[2] R. BOUARD and M. Coutanceau. The early stage of development of the wake behind an impulsively started cylinder for $40<\mathrm{R}_{e}<10000$. J. Fluid Mech., 101:583-607, 1980.

[3] T. SARPKAYA. Experiments on the stability of sinusoidal flow over a circular cylinder. J. Fluid Mech., 457:157-180, 2002.

[4] STAR-CD. Methodology, User Guide. CD Adapco, 2004.

[5] Z.U.A. WARSI. Conservation form of the navier-stokes equations in general non steady coordinates. AIAA Journal., 19:240-242, 1980.

[6] T.J.R. Hughes, W.K. LiU, and T.K. Zimmerman. Lagrangian eulerian finite element formulation for incompressible viscous flows. In US-Japan Seminar on Interdisciplinary Finite Element Analysis, New York, 1978.

[7] R.I. IsSA. Solution of the implicitly discretised fluid flow equation by operator splitting. J. Comput. Phys., 62:40-65, 1985.

[8] V. Melot, J.F. Sigrist, B. Auvity, C. Laine, and H. PEERHOSSAINI. Investigation of flow and forces on as strongly accelerated circular cylinder. In ASME Pressure Vessel and Piping Div. Conf, Denver, USA, 17-21 July 2005.

[9] R.J. FRITZ. The effects of liquids on the dynamics motion of immersed solids. Trans. ASME, J. Eng. Ind., pages 163$171,1972$.

[10] H. Schlichting. Boundary Layer Theory, $7^{\text {th }}$ Edition. McGraw-Hill, 1979.

[11] E. DeLangre. Fluides et Solides. Les éditions de l'Ecole Polytechnique, 2001.

[12] F. AXISA. Modélisation des systèmes mécaniques. Interactions fluide/structure. Hermès, 2001.

[13] M. VAnDy Ke. Perturbation Methods in Fluid Mechanics. Academic Press, New York and London, 1964.

[14] Y. LU and R.J. RoGERS. Instantaneous squeeze film force between a heat exchanger tube with arbitrary tube motion and a support plate. J. Fluids Struct., 9:835-860, 1995.

[15] T. ZHOU and R.J. ROGERS. Simulation of two-dimensional squeeze film and solid contact forces acting on a heat exchanger tube. J. Sound. Vib., 203:621-639, 1997.

[16] G. K. Batchelor. An Introduction to Fluid Dynamics. Cambridge University Press, Cambridge, 1997.

[17] V. Melot, J.F. Sigrist, B. Auvity, C. Laine, and H. PEERHOSSAINI. Fluid forces on a moving body at low amplitude in fluid at rest. part 1. a review of literature. In ASME Pressure Vessel and Piping Div. Conf, Vancouver, Canada, 23-27 July 2006.

[18] M. Abramowitz and I.A. Stegun. Handbook of Mathematical Functions. Dover, 1970.

[19] G.G. STOKES. On the effect of the internal friction of fluids on the motion of pendulums. Trans Camb Phil Soc., 9:8, 1851.

[20] L. ROSENHEAD. Laminar Boundary Layers: an account of the development, structure and stability of laminar boundary layers in incompressible fluids, together with a description of the associated experimental techniques. Oxford Univ. Press, 1963. 